\title{
Soft X-ray polarimeter-spectrometer SOLPEX
}

\author{
Marek Stȩślicki ${ }^{1}$, Janusz Sylwester ${ }^{1}$, Stefan Płocieniak ${ }^{1}$, Jarosław \\ Bạkała $^{1}$, Żaneta Szaforz ${ }^{1,2}$, Daniel Ścisłowski ${ }^{1}$, Mirosław Kowaliński ${ }^{1}$, \\ Jose Hernandez ${ }^{1}$, Sergey Kuzin ${ }^{3}$ and Sergey Shestov ${ }^{3}$ \\ ${ }^{1}$ Solar Physics Division, Space Research Centre Polish Academy of Sciences \\ Kopernika 11, 51-622 Wrocław, Poland \\ email: sm@cbk.pan.wroc.pl \\ ${ }^{2}$ Astronomical Institute, University of Wroclaw \\ Kopernika 11, 51-622 Wrocław, Poland \\ email: zs@cbk.pan.wroc.pl \\ ${ }^{3}$ P.N. Lebedev Physical Institute, Russian Academy of Sciences \\ Moscow, Russian Federation \\ email: kuzin@sci.lebedev.ru
}

\begin{abstract}
We present an innovative soft X-ray polarimeter and spectrometer SOLPEX. The instrument is to be mounted aboard the ISS within the Russian science complex KORTES. The measurements to be made by SOLPEX are expected to be of unprecedented quality in terms of sensitivity to detect the soft-X- ray polarization of solar emission emanating from active regions and flares in particular. Simultaneous measurements of the polarization degree and the other characteristics (eg. evolution of the spectra) constitute the last, rather unexplored area of solar $\mathrm{X}$-ray spectroscopy providing substantial diagnostic potential. Second important science task to be addressed are the measurements of Doppler shifts in selected X-ray spectral emission lines formed in hot flaring sources. The novel-type Dopplerometer (flat Bragg crystal drum unit) is planned to be a part of SOLPEX and will allow to measure line Doppler shifts in absolute terms with unprecedented time resolution (fraction of a second) during the impulsive flare phases. We shall present some details of the SOLPEX instrument and discuss observing sequences in a view of science objectives to be reached.
\end{abstract}

Keywords. solar, X-rays, spectroscopy, polarimetry

\section{Introduction}

Polarimetry of solar flares is a powerful diagnostic of the properties of the magnetic field and of the hot plasma in the atmosphere of our star. Flares are thought to be generated by magnetic reconnection that produces a sudden release of energy stored in the magnetic field configuration and gives rise to violent particle acceleration. Non-thermal hard X-ray (HXR) emission is produced as a result of electrons slowing down in the plasma (bremsstrahlung emission). Models assuming anisotropic distributions of accelerated electrons in an ordered magnetic field predict that this component should be highly polarized, with a polarization degree as high as $40 \%$ at $20 \mathrm{keV}$ (Zharkova et al., 2010). Instead, soft X-ray emission (at energies $<10 \mathrm{keV}$ ) is generated by plasma heating taking place in the reconnection site and along the flare loop and it is characterized by lines' emission up to about $7 \mathrm{keV}$. This component is also expected to be polarized, although at a lower level than HXR, due to possible anisotropies in the electron distribution function (Emslie \& Brown, 1980). Moreover, the backscattering of radiation on lower levels 
of solar atmosphere can modify polarization properties of reflected radiation (Jeffrey \& Kontar, 2011).

The first measurements intended to measure the X-ray polarization from the solar flares were placed on-board Intercosmos satellites and indicated for rather high average polarization $\mathrm{P}=40 \%$ (Tindo et al., 1970) and 20\% (Tindo et al., 1972a, 1972b) at energies $\sim 15 \mathrm{keV}$. Subsequent observations carried on-board OSO-7 satellite showed lover magnitudes of the polarization $10 \%$ (Nakada et al., 1974) in this range of energies. A later measurements using instrument on-board Intercosmos 11 showed polarization of only few percent at this energies (Tindo et al., 1976). It is consistent with measurement obtained using polarimeter on-board STS-3 mission which showed upper limits in the range of $2.5 \%$ to $12.7 \%$ in the $5-20 \mathrm{keV}$ energy range (Tramiel et al., 1984). The most recent measurements of the solar flares X-ray flux polarization at higher energies were obtained by two instruments: RHESSI and SPR-N polarimeter on-board the CORONAS-F satellite.

Using RHESSI data McConnell et al. (2003) obtained the polarization of $18 \%$ in the energy range $20-40 \mathrm{keV}$ from the solar flare of SOL2002-07-23T00:35. At even higher energies $(0.2-1 \mathrm{MeV})$ polarization of $21 \% \pm 9 \%$ and $11 \% \pm 5 \%$ were measured by Boggs et al. (2006) for the same event and other X-class flare (SOL2003-10-28T11:10). SuarezGarcia et al. (2006) found values for the polarization degree in the range between $2 \%$ and $54 \%$, with statistical errors from $10 \%$ to $26 \%$ at the $1 \sigma$ level, at energy range from 100 to $350 \mathrm{keV}$, for six X-class and one M-class flares from the RHESSI database.

Zhitnik et al. (2006) presented results of observations from the SPR-N polarimeter on-board the CORONAS-F satellite. Among 90 analyzed flares one event (X10 flare, SOL2003-10-29T20:49) showed a significant polarization degree exceeding $70 \%$ in channels of $\mathrm{E}=40-60$ and $60-100 \mathrm{keV}$ and about $50 \%$ in the 20 to $40 \mathrm{keV}$ channel. For 25 events, the upper limits of the part of polarized X-rays were estimated at the levels between $8 \%$ and $40 \%$.

Unfortunately, every of these instruments did not obtain high significance results to address this polarisation properties of X-ray emission. The set of instruments planned as the SOLPEX block for the ISS, in particular the B-POL Bragg polarimeter unit can provide unique opportunity to complement the efforts to reliably measure polarization at lower end of non-thermal energies and thus contribute towards better understanding the physics of solar flares. The standard flare model states that electrons are being accelerated at or near of the magnetic reconnection site in specific regions of the corona and then propagate along newly reconnected magnetic field lines towards the atmospheric denser layers. Here, they are decelerated by the increasingly growing "opposition" of the atmosphere and lose their energy mainly through the bremsstrahlung process. Deposited energy is readily converted to directed evaporation of the plasma to be observed through the bulk motion that has traditionally been detected though Doppler measurements of extreme ultraviolet and soft X-ray emission lines (eg. Milligan \& Dennis, 2009, Battaglia et al., 2015). Both those processes: bremsstrahlung emission of supposedly polarized Xray flux and corresponding evaporation velocities can be simultaneously observed by the proposed SOLPEX set of instruments.

\section{The instrument}

The planned set of measurement blocks, which make up the the SOLPEX, creates a unique opportunity to obtain accurate measurements of the polarization degree and direction of polarisation plane at the region of formation of a soft X-ray radiation in solar flares.

SOLPEX instrument in itself will be a part of the Kortes instruments complex that will 


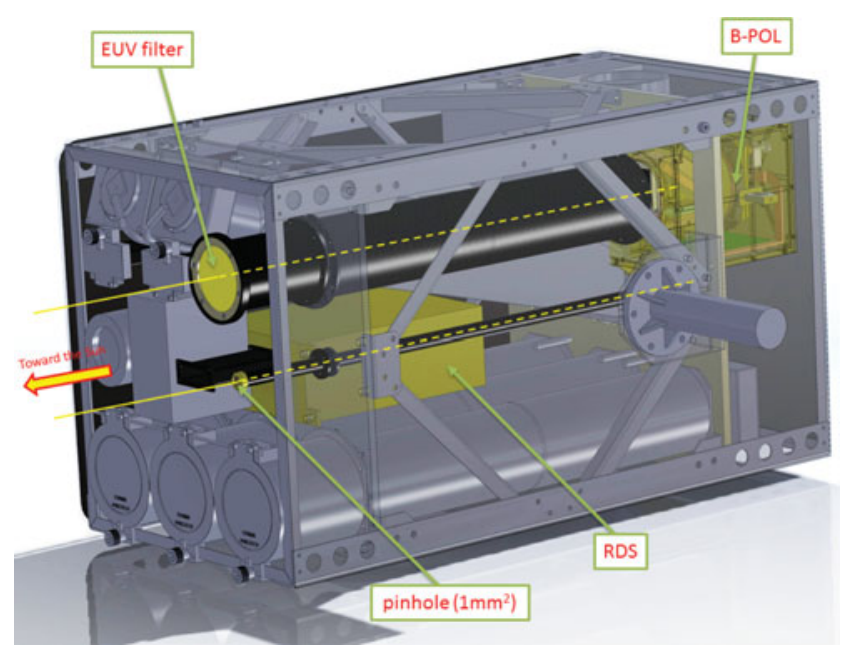

Figure 1. Kortes platform with SOLPEX modules marked.

be placed on-board the International Space Station (ISS), on a solar-pointed platform. The main scientific objectives of the SOLPEX instrument are:

- Measurement of the linear polarization of the X-ray radiation originating in solar flares, as well as other astrophysical sources which are within observation.

- Examine changes in the physical parameters of a flaring plasma. This reletes to: distribution of plasma temperatures within flaring volume, so called DEM, changes of the turbulent velocity, identification of spectral lines Doppler shifts and determination of the chemical composition of the emitting plasma.

- Measurement of the X-ray solar spectrum of in the range 1-15 keV, with an moderate spectral resolution, and high time resolution of close to $1 \mathrm{~ms}$, with a sensitivity of about $10^{-10} \mathrm{~W} / \mathrm{m}^{2}$.

- Measurements of solar flares and active regions X-ray flux with a very high temporal resolution and unprecedented spectral resolution.

All those measurements will be performed with unprecedented temporal resolution.

SOLPEX instrument consists of three partially independent modules (Fig. 1) arranged within the Kortes platform:

\subsection{Rotating drum spectrometer $(R D S)$}

RDS is the fast rotating drum spectrometer with large crystals. This instrument module will measure rapid changes in spectral lines intensity and it's Doppler shifts. The design of this block is novel. This module consists of the octagonal drum with eight flat crystals attached. The drum rotates at a frequency of 10 revolutions per second. Light emitted by the solar corona is reflected by rotating crystals according to the Bragg laws and illuminates four silicon drift detectors (SDD) - Fig. 2. In this module Ketek Vitus R100 detectors will be used. These detectors have a large effective window area and fast response time $(1 \mu \mathrm{s})$. The dynamic range of the instrument is greater than $10^{5}$. The RDS uses an innovative method to assign the Bragg angle to each photon being registered by the detector. At the time of registration, known to an accuracy of $1 \mu \mathrm{s}$, the detector and the crystal angle can be easily determined with accuracy of approx. 10 arcseconds, which is smaller then the width of reflection curve. In this way, for each crystal a counts histogram per unit angle or wavelength can be constructed by the on-board computer. Depending on the intensity of the source the number of counts required for the integration 
Table 1. Crystals for RDS X-ray spectrometer. For each crystal, the two spectral waveranges are presented. First wavelength band is for front pair of detectors (two detectors being closer to the window). Second waverange is for the back pair of SDD detectors.

\begin{tabular}{lccccc}
\hline No & Crystal & Orientation & $\begin{array}{l}\mathbf{2 d} \\
{[\AA]}\end{array}$ & $\begin{array}{c}\text { Detector } \mathbf{1} \\
\text { wavelength range }[\AA]\end{array}$ & $\begin{array}{c}\text { Detector 2 } \\
\text { wavelength range }[\AA]\end{array}$ \\
\hline 1. & $\mathrm{Si}$ & 400 & 2.715 & $1.397-2.331$ & $0.27-1.796$ \\
2. & $\mathrm{Si}$ & 220 & 3.840 & $1.977-3.298$ & $0.391-2.541$ \\
$3 .^{\dagger}$ & $\mathrm{Si}$ & 111 & 6.271 & $3.228-5.385$ & $0.639-4.150$ \\
4. & Quartz & $10-11$ & 6.684 & $3.441-5.740$ & $0.681-4.423$ \\
5. & Quartz & $10-10$ & 8.514 & $4.383-7.312$ & $0.868-5.635$ \\
6. & $\mathrm{ADP}$ & 101 & 10.648 & $5.482-9.145$ & $1.086-7.047$ \\
7. & KAP & 001 & 26.640 & $13.717-22.880$ & $2.718-17.631$ \\
\hline
\end{tabular}

Note: ${ }^{\dagger}$ Two pieces of this crystal arranged in Dopplerometer configuration will be used.

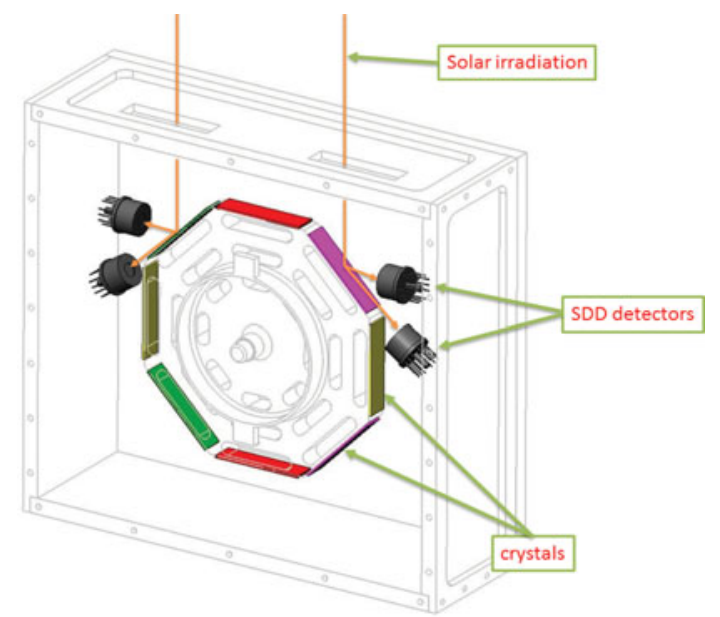

Figure 2. RDS scheme. Eight crystals attached to the rotating drum and four SDD detectors (two on each side of the drum) are featured.

of the spectra is reached within minutes (for an active region) or less then a second (for a flare classes above M5).

The spectrometer will be equipped with seven different crystals. Additionally each crystal will reflect incoming photons according to the Bragg towards the two differently positioned SDD detectors. This setup allows to cover wider range of energies (Table 1). Two of the eight crystals mounted on the drum are identical and arranged in a doplerometer configuration (Sylwester et al., 2015). Determination of the presence and amount of the Doppler shifts in the spectra lines is one of the main objectives of the SOLPEX. These shifts contain the information about the evaporation rate of the plasma during the impulsive phase of the flare. This quantity will be measured with a few seconds time resolution for stronger events.

\subsection{Bragg Polarimeter (B-POL)}

The instrument will measure the degree of linear polarization of solar flares impulsive phase in the soft X-ray range. Measurements will be carried out in a narrow range of the spectrum by using a bend silicon monocrystal wafer, with reflective plane 111 and a curvature radius of $610.0 \mathrm{~mm}$. The reflected spectrum will be recorded by a large area CCD detector. A pair of crystal-CCD will rotate around the axis directed at a solar flare, with the angular speed of of 1 revolation/sec (Fig. 3). The spectrum recorded by 


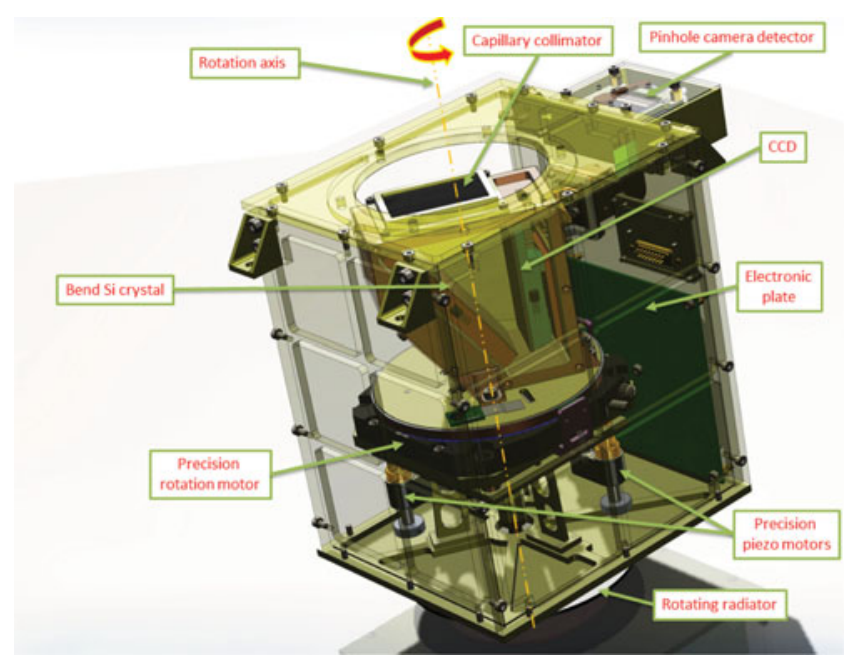

Figure 3. Outline of B-POL polarimeter.

the detector will be read every $30^{\circ}$ of the rotation, i.e., at least 12 times per second. The crystal is chosen so that the spectrum is measured in the vicinity of the so-called Brewster angle $\left(\sim 45^{\circ}\right)$ wherein the reflection efficiency depends strongly on the degree of linear polarization of the incoming radiation. In the extreme case, when the degree of linear polarization of the incident radiation is $100 \%$ a modulation level also will reach $100 \%$ (at the wavelength corresponding to the Brewster angle). The existence of the modulation of spectrum in line intensities and continuum during revolution indicate the presence of polarization. The modulation depth contains information on the degree of polarization, and maximum/minimum modulation phases determine the position of the plane of polarization with respect to the solar coordinates. To limit the field of view to one active region $(\sim 2 \times 2$ arcminutes) polarimeter B-POL will be equipped with a capillary collimator covering the entire crystal illumination area. The rotation axis of B-POL will be pointed at the flaring active region. Information about the brightness and location of the flare will be passed by the on-board computer based on X-ray "on-line" image analysis of the whole solar disc, which will be continuously supplied from the pinhole camera system.

\subsection{The pinhole imager (PHI)}

The pinhole camera is made of the round hole $\left(\sim 1 \mathrm{~mm}^{2}\right)$ and CCD detector (E2V CCD30$11,256 \times 1024$ pixels), which is located at a distance of about $60 \mathrm{~cm}$. In front of the pinhole carbon filter is located that transmits only the photons with energies above $\sim 0.5 \mathrm{keV}$. Radiation at these energies is emitted by both active regions and flares, as well as in this range the limb brightening effect is observed. Analysis of the limb brightening position will determine of the location of active regions on the solar disk. Images will be taken and analyzed several times a second on a continuous basis, which will help to determine the moment of the beginning of the flare and make it possible to direct the polarimeter rotation axis at a brightest point before the advent of the impulsive phase.

\section{Summary}

The B-POL Bragg polarimeter unit will provide unique opportunity to complement the efforts to reliably measure polarization at lower end of non-thermal energies. Additionally 
the RDS spectrometer will simultaneously measure the soft X-ray spectra and plasma Doppler shifts. Therefore the planed set of modules will contribute towards understanding the physics of solar flares.

We estimate, taking into an account the ISS environment, that during it's operation the instrument will observe about 50 C-class, $5 \mathrm{M}$ class, and one X-class flare.

\section{Acknowledgements}

We acknowledge financial support from the Polish National Science Centre grant 2013/11/B/ST9/00234.

\section{References}

Battaglia, M., Kleint, L., Krucker, S., \& Graham, D. 2015, ApJ, 813, 113

Beiersdorfer, P., Vogel, D. A., \& Reed, K. J. et al. 1996, Physical Review A, 53, 3974

Boggs, S. E., Coburn, W., \& Kalemci, E. 2006, ApJ, 638, 1129

Emslie, A. G. \& Brown, J. C. 1980, ApJ, 237, 1015

Jeffrey, N. L. S. \& Kontar, E. P. 2011, A\&3 A, 536, 93

Lin, R. P. 2011, Space Science Reviews, 159, 421

McConnell, M. L. \& Smith, D. M., Emslie et al. 2003, Proc. SPIE, 4843, 1382

Milligan, R. O. \& Dennis, B. R. 2009, ApJ, 699, 968

Nakada, M. P., Neupert, W. M., \& Thomas, R. J. 1974, Solar Physics, 37, 429

Tramiel, L. J., Novick, R., \& Chanan, G. A. 1984, ApJ, 280, 440

Suarez-Garcia, E., Hajdas, W., \& Wigger, C et al. 2006, Solar Physics, 239, 149

Sylwester, J., Kordylewski, Z., \& Płocieniak, S. et al. 2015, Solar Physics, 239, 149

Tindo, I. P., Ivanov, V. D., Mandel'Stam, S. L., \& Shuryghin, A. I. 1970, Solar Physics, 14, 204

Tindo, I. P., Ivanov, V. D., Mandel'Stam, S. L., \& Shuryghin, A. I. 1972a, Solar Physics, 24, 429

Tindo, I. P., Ivanov, V. D., Valníček, B., \& Livshits, M. A. 1972b, Solar Physics, 27, 426

Tindo, I. P., Shurygin, A. I., \& Steffen, W. 1976, Solar Physics, 46, 219

Zharkova, V. V., Kuznetsov, A. A., \& Siversky, T. V. 2010, A\& A, 512, 490

Zhitnik, I. A., Logachev, Yu.I., Bogomolov, A. V., \& Denisov, Yu. et al. 2006, Sol. Sys. Res., 40,93 